\title{
Brain tumors and indications for brain imaging in patients with psychiatric manifestations: a case report
}

\author{
Seyed Alireza Haji Seyed Javadi and Bahare Rezaei* (D)
}

\begin{abstract}
Background: Studies on the relationship between psychiatric symptoms and brain tumors are ambiguous, as it is not clear whether these symptoms are due to the direct effect of the tumor or a secondary psychological response to stress, resulting from the diagnosis and treatment of the disease; therefore, it is difficult to analyze and retrieve relevant information.

Case presentation: We present the case of a 43-year-old male patient, who was admitted to a psychiatric emergency room with psychiatric symptoms, such as restlessness and extreme talkativeness, but normal neurological examinations. He showed no response to outpatient treatment and had no history of psychiatric disorders. The onset of symptoms was 2 months before his visit. On neuroimaging, a brain tumor was observed in the right temporal and occipital lobes. Accordingly, the patient was transferred to the neurosurgery ward.

Conclusion: Factors, such as increased internal pressure on the brain due to a brain tumor or the effect of tumor area, contribute to the occurrence of symptoms, such as restlessness and talkativeness. However, further studies are needed to confirm these findings.
\end{abstract}

Keywords: Brain mass, Case report, Psychiatric symptoms, Restlessness

\section{Background}

Patients with brain tumors may have no neurological symptoms; therefore, psychiatric symptoms can be the only clue to a brain tumor in some cases [1]. Studies have shown that brain tumors may lead to psychiatric symptoms and behavioral changes [2,3]. Changes in the mental status may be the first sign of brain tumors. Therefore, neuroimaging is recommended not only for patients with neurological symptoms but also for patients whose psychiatric symptoms have changed or have abnormal symptoms $[4,5]$.

Psychiatric symptoms, such as abulia, apathy, anxiety, depression, psychosis, mania, and personality and

\footnotetext{
* Correspondence: bahare.rezaei1363@gmail.com

Clinical Research Development Unit, 22 Bahman Hospital, Qazvin University of Medical Sciences, Qazvin, Iran
}

\section{Springer Open}

cognitive changes, are seen in patients with brain tumors [6]. Various studies have described the effects of tumor location on the type of symptoms. For example, a dorsolateral prefrontal cortex tumor is associated with problems in individual organization and planning; orbitofrontal tumors cause disinhibition; and middle forehead tumors are linked to apathy and abulia [7]. Restlessness is one of the lesser-known symptoms associated with brain tumors $[8,9]$; involvement of different parts of the brain may cause this symptom. Overall, restlessness may occur frequently in different stages of tumor and cancer development $[8,9]$.

Since the rate and duration of tumor growth can affect the severity and course of symptoms, the location of the tumor is often associated with the symptoms [10]. Studies of psychiatric symptoms and their association with brain tumors are ambiguous because it is not clear whether the symptoms are tumor-induced or a

(c) The Author(s). 2021 Open Access This article is licensed under a Creative Commons Attribution 4.0 International License, which permits use, sharing, adaptation, distribution and reproduction in any medium or format, as long as you give appropriate credit to the original author(s) and the source, provide a link to the Creative Commons licence, and indicate if changes were made. The images or other third party material in this article are included in the article's Creative Commons licence, unless indicated otherwise in a credit line to the material. If material is not included in the article's Creative Commons licence and your intended use is not permitted by statutory regulation or exceeds the permitted use, you will need to obtain permission directly from the copyright holder. To view a copy of this licence, visit http://creativecommons.org/licenses/by/4.0/. 
secondary psychological response to post-diagnosis or treatment stress. Besides, the descriptions of psychiatric symptoms in review studies are not similar $[1,10]$. Therefore, the lack of extensive studies has made this clinical conundrum a complex one, especially regarding restlessness.

Here, we report the case of a 43-year-old man, who presented to a psychiatric emergency department with symptoms of restlessness and extreme talkativeness, but normal neurological examinations without response to outpatient treatment. These changes in the mental status, without neurological symptoms on brain neuroimaging, were diagnosed as a brain tumor.

\section{Case presentation}

The patient was a 43-year-old man with severe restlessness, who was referred to the psychiatric ward of 22 Bahman Hospital in Qazvin Province, Iran. According to the patient's description, the onset of symptoms was about 2 months before his visit to our hospital. At first, the patient had experienced restlessness and sleep disturbances, especially early morning awakening. According to his description, his restlessness increased as soon as he woke up, and he started asking others for help. The patient's companions believed that he had become talkative over the past 2 months and that in his speech, he was asking for help due to restlessness. The patient was restless at the time of his visit and was walking constantly. Examination of his mental status revealed that he had an anxious mood, but there was no specific pathological finding in his thought patterns.

One week before admission, the patient was referred to a psychiatrist and treated with medications, including fluoxetine $(20 \mathrm{mg})$, amitriptyline (25 $\mathrm{mg}$ every night), risperidone $(1 \mathrm{mg})$, propranolol $(40 \mathrm{mg}$; half a tablet in the morning, and half a tablet at night), and chlordiazepoxide $(10 \mathrm{mg}$; one tablet in the morning and one at night). However, no improvement was seen in his symptoms. One month before his admission, a history of impotence was found in the patient's clinical history, which did not improve with sildenafil. There was no major finding in the patient's psychiatric history or history of medication use, especially in terms of hypnotic, antimuscarinic, and anticonvulsant medications. The patient was also examined for substance use, such as opioids, methamphetamine, and cannabis, but no substance use disorder was observed. Besides, there was no considerable finding in the patient's family history.

\section{Mental status examination}

In the mental status examination, the patient's attitude was semi-cooperative. In terms of psychomotor activity, restlessness, high speech volume, spontaneous speech, anxious mood, and mood congruence effect were reported. Except for impaired concentration, no specific pathological finding was reported in the cognitive assessments or the Mini-Mental State Examination (MMSE).

\section{Neurological examinations}

The cranial nerve examination was normal. In the motor examination, the muscle volume and tone also were normal. The muscle strength was found to be $5 / 5$ (the muscle worked normally and could maintain its position). Also, examinations of the sensory system, abdominal, plantar, and deep tendon reflexes were normal. No significant findings were found in the ophthalmoscopic examination. The patient's gait and cerebellar examinations were also normal.

Moreover, the patient's vital signs were completely normal. At the onset of hospitalization, necessary tests were performed to rule out medical disorders associated with restlessness, such as fever, infection, and electrolyte disorders (i.e., a pathological increase in serum calcium). To start medications, fasting blood sugar (FBS) tests, lipid profile, liver function test, thyroid function test, electrolytes (i.e., sodium, potassium, calcium, and phosphorus), creatinine, blood urea nitrogen (BUN), complete blood count (CBC), urine toxicology, and electrocardiogram (ECG) were requested, all of which were reported to be normal.

Next, the patient was asked to undergo a brain CT scan, which was not performed on the first day of hospitalization due to his severe restlessness and was postponed until it was managed. lorazepam was prescribed to control the patient's restlessness, which was changed to clonazepam ( $1 \mathrm{mg}$ in the morning, $1 \mathrm{mg}$ in the afternoon, and $2 \mathrm{mg}$ at night) due to the ineffectiveness of lorazepam. Medications, including Rahakin (sodium valproate, $500 \mathrm{mg}$ twice daily), olanzapine (5 mg twice daily), and propranolol (10 mg three times daily), were prescribed, with an initial diagnosis of bipolar disorder not otherwise specified (NOS). His restlessness was partially controlled 2 days after receiving the drug combination, but he developed ataxia. In the meantime, a brain CT scan was performed.

After performing CT scan and observing the mass, the patient underwent magnetic resonance imaging (MRI), as suggested by the neurosurgeon; a large mass was detected in the right temporal and occipital lobes. Figure 1 $\mathrm{a}, \mathrm{b}$, and $\mathrm{c}$ show the axial and coronal views of the T2weighted MRI image of the brain without contrast. The tumor dimensions were $100 \times 60 \times 50 \mathrm{~mm}$, as can be seen in the right temporal and occipital lobes. This tumor had compressed the third ventricle, midbrain, and right lateral ventricle, shifting the midline elements and enlarging the left lateral ventricle (Fig. 1). Consequently, the patient was transferred to the neurosurgery service. 


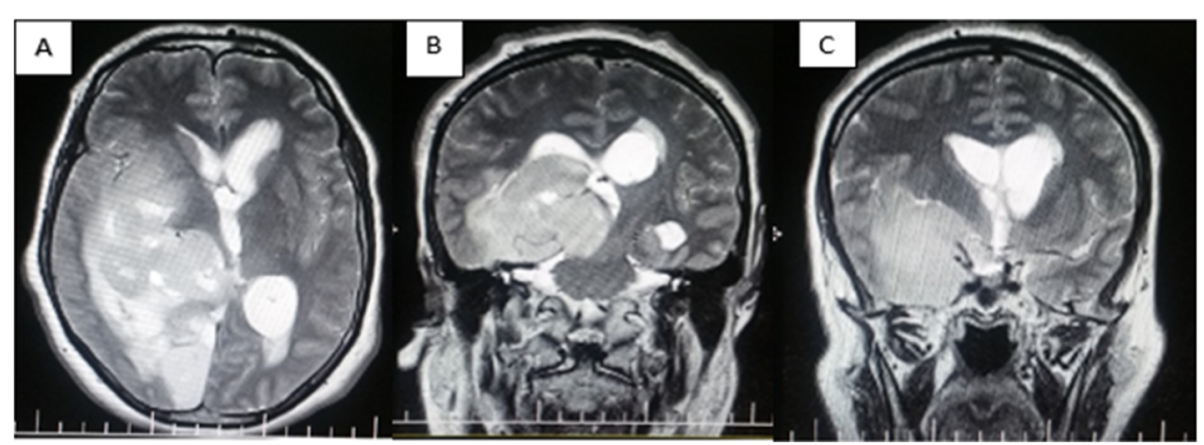

Fig. 1 a, b, c The axial and coronal views of T2-weighted MRI of the brain without contrast. The dimensions of the tumor are $100 \times 60 \times 50$ mm in the right temporal and occipital lobes, compressing the third ventricle, midbrain, and right lateral ventricle, causing the shift of midline elements and enlargement of the left lateral ventricle

\section{Discussion}

Based on our literature review, we categorized psychiatric symptoms according to the location of brain tumor $[10,11]$. This study, by reporting another case, aimed to promote research in the field of psychiatry. The results showed that there might be a significant association between the tumor location in the temporal and occipital lobes and restlessness as a symptom. In this regard, some studies have reported a significant association between anorexia nervosa and hypothalamic tumors. Also, some studies have shown that there is a possible association between psychotic symptoms and pituitary tumors; between memory symptoms and thalamic tumors; and between mood symptoms and forehead tumors.

Psychiatric symptoms have been observed in various types of diseases related to the nervous system, including brain tumors $[3,10]$. Therefore, accurate history-taking is essential when discussing brain tumors in patients with psychiatric symptoms [1], because it allows the physician to determine the underlying cause and choose an appropriate treatment according to the patient's clinical symptoms. Studies have shown that infections and fever play an important role in restlessness $[8,12]$. However, preliminary examinations showed no such symptoms in our patient's medical history.

In this case report, to evaluate the side effects of used medications on restlessness and talkativeness, the patient's medical records were reviewed. Generally, research shows that many medications used in palliative care may cause some degree of stimulation and restlessness. Sudden discontinuation of hypnotics, antimuscarinic drugs, and anticonvulsants has been shown to have such effects [13, 14]; however, no side effects or drug interactions were observed in our patient's medical history.

The patient was also evaluated for the use of substances, such as methamphetamine, cannabis, and opioids, as the sudden use or withdrawal of some substances can also lead to neurostimulation and restlessness [15]. These initial examinations were important to determine the association of each of these factors with restlessness. Hypercalcemia may also play a role in causing restlessness [16]. It is the most common life-threatening metabolic disorder in cancer patients, which can cause restlessness and confusion; however, there was no evidence of hypercalcemia in our patient.

In another study of eight patients, the cause of referral to the clinic was only psychiatric symptoms; these patients underwent psychiatric treatment until the onset of neurological symptoms. Early diagnoses included depression, anxiety, schizophrenia, anorexia nervosa, and cognitive problems. Brain tumors were diagnosed after the onset of neurological symptoms by performing brain imaging. It seems that imaging should not be considered only in patients with neurological symptoms but also in patients with purely psychiatric, atypical, or changing symptoms or those whose symptoms do not correspond to the age of onset [17]; such observations are of great importance for timely diagnosis and treatment [17].

So far, few studies have been conducted on the association of restlessness with brain tumors [8]. This study aimed to contribute to the current literature by reporting a case. We concluded that pathologies in the temporal and occipital regions may play a prominent role in the pathogenesis of restlessness due to the involvement of these regions and the compressive effect of tumor on the basal ganglia, especially in the right hemisphere. This finding is consistent with previous studies, which suggested that abnormalities in the basal ganglia and their association with the cortex and brainstem play a role in the pathophysiology of hyperkinesia and psychomotor disorders; they suggested a similar brain mechanism in the pathogenesis of restlessness and its treatment [18, 19].

In another case series, a patient was hospitalized with symptoms of restlessness, headache, vomiting, and unrelated speech. Evaluations performed by physicians revealed that the patient had a glioblastoma tumor in the 
frontal region [9]. Another study examined the association of mania symptoms in patients with brain tumors and reported that most of these tumors were on the right side of the brain, including the frontal and temporal regions, as well as the limbic cortex structures; this finding is consistent with the present study in terms of tumor location and symptoms, such as talkativeness [20].

Based on the present findings, there were no medical or neurological symptoms related to the size of tumor in our patient. Regarding the involvement of temporal and occipital lobes and the compressive effect of tumor on the basal ganglia, especially in the right hemisphere, pathologies in these areas may play a prominent role in the pathogenesis of restlessness. There may be several factors contributing to this phenomenon. Previous research shows that restlessness might be associated with biochemical abnormalities in the body; these abnormalities occur following the onset of organ failure (especially tumors) [8]. Another reason will be the increased pressure of the brain tumor. Research shows that tumors can increase the intracranial pressure and stimulate the patient, depending on the area involved [10].

The limitation of this study is that longitudinal followup of the patient after referral to another hospital for neoadjuvant chemotherapy was not possible. The strength of this study is the robust system of recording the patient's information, which enabled us to extract his information.

\section{Conclusions}

This case report showed that a patient with psychiatric manifestations, despite the absence of neurological symptoms, had a large brain tumor. Therefore, the compressive effect of tumor on the midline elements and the third ventricle of the brain in the absence of neurological symptoms should be considered. Besides, the present findings showed that neuroimaging for patients with such psychiatric symptoms can be very important in the rapid diagnosis of disease. Factors, such as increased internal pressure on the brain due to brain tumor and tumor location, may be involved in the development of these symptoms (restlessness and talkativeness). To confirm this finding, basic studies and further research are needed.

\section{Abbreviations}

FBS: Fasting blood sugar; LFT: Liver function test; TFT: Thyroid function test; BUN: Blood urea nitrogen; CBC: Complete blood count;

ECG: Electrocardiogram; CT: Computed tomography; NOS: Not otherwise specified; MRI: Magnetic resonance imaging

\section{Acknowledgements}

We deeply appreciate the collaboration of Dr. Ali Akbar Shafiakhani and Dr. Samira Dodangeh in Qazvin Research Group for editing this manuscript.

\section{Authors' contributions}

I have the pleasure of sending you the manuscript entitled "Brain tumors and indications for brain imaging in patients with psychiatric manifestations: A case report" authored by SAH and BR to be considered for publication as a research article in your prestigious journal. All authors have seen and approved the manuscript and have contributed significantly for the paper, and ensure that this is the case. SAH: visualization, conceptualization, investigation, data curation, and writing, reviewing, and editing the manuscript. BR: Conceptualization, visualization, investigation, supervision, and writing of the original draft.

\section{Funding}

The authors declare that they have no funding/financial sources.

\section{Availability of data and materials}

All data generated and analyzed during this study are included in this published article.

\section{Declarations}

Ethics approval and consent to participate

This study was approved by the Ethics Committee of Qazvin University of Medical Sciences (Ethic Code: IR.QUMS.REC.1400.063). A written consent form was also obtained from the patient for this study.

\section{Consent for publication}

Written informed consent was obtained from the patient for publication of this case report and accompanying images.

\section{Competing interests}

The authors declare that they have no competing interests.

Received: 16 June 2021 Accepted: 13 August 2021

Published online: 01 October 2021

\section{References}

1. Agrawal N, Faruqui R, Bodani M (2020) Oxford textbook of neuropsychiatry. United Kingdom: Oxford University Press. https://doi.org/10.1093/med/ 9780198757139.001.0001

2. Farzaneh R, Malek A, Mirzaei F, Amiri S, Salehpour F, Meshkini A, Musavi Z, Farhang S, Dastgiri S, Farzane A, Ghanbari F (2020) Evaluation of comorbid psychiatric disorders in patients with primary brain tumors before and after surgery. Neurol, Psychiatry Brain Res 36:8-13. https://doi.org/10.1016/j.npbr.2 020.02 .003

3. Madhusoodanan S, Opler MG, Moise D et al (2010) Brain tumor location and psychiatric symptoms: is there any association? A meta-analysis of published case studies. Expert Rev Neurother 10(10):1529-1536. https://doi. org/10.1586/ern.10.94

4. Al-Harthy H, Al-Mahrouqi T, Al-Obaidani A, Al-Hashmi S, Mirza H (2020) Solitary Fibrous Tumor Revealed by Worsening Clinical Depression: A Case Report from Oman. Cureus 12(8):e9584. https://doi.org/10.7759/cureus.9584

5. Gonzalez R, Nemeroff CB (2020) Inflammatory diseases and their psychiatric manifestations, in Textbook of Medical Psychiatry. Washington, DC: American Psychiatric Association Publishing. p. 229-263

6. Moise D, Madhusoodanan S (2006) Psychiatric symptoms associated with brain tumors: a clinical enigma. CNS Spectr 11(1):28-31. https://doi.org/10.1 017/S1092852900024135

7. Litofsky NS, Resnick AG (2009) The relationships between depression and brain tumors. J Neurooncol 94(2):153-161. https://doi.org/10.1007/s11060009-9825-4

8. McCain J, Salter M (2019) The restless wave: Good times, just causes, great fights, and other appreciations. New York; Simon \& Schuster

9. Rohatgi S (2020) Eye as a window to glioblastoma multiforme: a series of case reports. Med J Dr DY Patil Vidyapeeth 13(2):184. https://doi.org/10.41 03/mjdrdypu.mjdrdypu_283_19

10. Subramoniam Madhusoodanan MBT, Farah T, Ugur U (2015) Psychiatric aspects of brain tumors: a review. World J Psychiatry 5(3):273-285. https:// doi.org/10.5498/wjp.v5.i3.273

11. Gyawali S, Sharma P, Mahapatra A (2019) Meningioma and psychiatric symptoms: an individual patient data analysis. Asian J Psychiatr 42:94-103. https://doi.org/10.1016/j.ajp.2019.03.029 
12. Jeon MS, Agar MR, Koh ES, Nowak AK, Hovey EJ, Dhillon HM (2021) Understanding sleep disturbance in the context of malignant brain tumors: a qualitative analysis. Neurooncol Pract 8(2):179-189. https://doi.org/10.1093/ nop/npaa081.

13. Khushboo SB, Sharma B (2017) Antidepressants: mechanism of action, toxicity and possible amelioration. J Appl Biotechnol Bioeng 3:1-13

14. Reyad AA (2018) Pharmacological alternatives to antipsychotics in the management of BPSD

15. Cutrufello NJ, lanus VN, Rowley JA (2020) Opioids and sleep. Curr Opin Pulm Med 26(6):634-641. https://doi.org/10.1097/MCP.0000000000000733

16. Shrimanker I, Bhattarai S (2020) Electrolytes. StatPearls. Treasure Island (FL): StatPearls Publishing.

17. Bunevicius A, Deltuva VP, Deltuviene D, Tamasauskas A, Bunevicius R (2008) Brain lesions manifesting as psychiatric disorders: eight cases. CNS Spectr 13(11):950-958. https://doi.org/10.1017/\$1092852900014000

18. Liberg B, Rahm C (2015) The functional anatomy of psychomotor disturbances in major depressive disorder. Front Psych 6:34

19. Fox MD (2018) Mapping symptoms to brain networks with the human connectome. N Engl J Med 379(23):2237-2245. https://doi.org/10.1056/ NEJMra1706158

20. Satzer D, Bond DJ (2016) Mania secondary to focal brain lesions: implications for understanding the functional neuroanatomy of bipolar disorder. Bipolar Disord 18(3):205-220. https://doi.org/10.1111/bdi.12387

\section{Publisher's Note}

Springer Nature remains neutral with regard to jurisdictional claims in published maps and institutional affiliations.

\section{Submit your manuscript to a SpringerOpen ${ }^{\circ}$ journal and benefit from:}

- Convenient online submission

- Rigorous peer review

- Open access: articles freely available online

- High visibility within the field

- Retaining the copyright to your article

Submit your next manuscript at $\boldsymbol{\nabla}$ springeropen.com 